\title{
Utilização das Curvas de Crescimento Intergrowth-21st para Recém-Nascidos Pré- Termo em uma Unidade de Terapia Intensiva Neonatal no sul do Brasil
}

The utilization of Intergrowth-21st Growth Curves for Preterm Newborns in a Brazilian Neonatal

\author{
Intensive Care Unit in southern Brazil \\ Uso de curvas de crecimiento Intergrowth-21st para recién nacidos prematuros en una unidad de \\ cuidados intensivos neonatales en el sur de Brasil
}

Recebido: 24/01/2021 | Revisado: 26/01/2021 | Aceito: 28/01/2021 | Publicado: 06/02/2021

\author{
Rafael de Oliveira Arrieira \\ ORCID: https://orcid.org/0000-0001-8404-1974 \\ Universidade Católica de Pelotas, Brasil \\ E-mail: rafaarrieira05@hotmail.com \\ Fernando Celso Lopes Fernandes de Barros \\ ORCID: https://orcid.org/0000-0001-5973-1746 \\ Universidade Católica de Pelotas, Brasi \\ E-mail: fcbarros.epi@gmail.com \\ Márcia Kaster Portelinha \\ ORCID: https://orcid.org/0000-0003-3649-6557 \\ Universidade Federal de Pelotas, Brasil \\ E-mail: portelinhamarcia@gmail.com
}

\begin{abstract}
Resumo
Utilizar as curvas de crescimento pós-natais do projeto INTERGROWTH-21st para recém-nascidos pré-termo internados na Unidade de Terapia Intensiva Neonatal do Hospital Escola da Universidade Federal de Pelotas-RS. Foram acompanhados 70 recém-nascidos, com delineamento longitudinal prospectivo, desde o nascimento e/ou internação até a alta ou óbito da unidade de terapia intensiva neonatal, no período de outubro de 2018 a abril de 2019. A idade gestacional, estimada variou de 19 a 36 semanas (média de 31 semanas), e $40 \%$ dos recém-nascidos tiveram peso ao nascer inferior a $1500 \mathrm{~g}$, com $89 \%$ apresentando peso inferior a $2500 \mathrm{~g}$. Sessenta recém-nascidos pré-termo tiveram alta e dez faleceram durante a hospitalização - mortalidade neonatal de 143 por 10000 nascidos vivos. Cerca de $60 \%$ dos nascimentos ocorreram por cesariana, e 13\% dos recém-nascidos apresentaram escore de Apgar abaixo de 4 no primeiro minuto de vida. Observou-se que através das medidas antropométricas, tanto em meninos quanto em meninas, durante a hospitalização, estiveram, em sua maioria, dentro dos limites normais de crescimento, sendo que os percentis de crescimento das crianças que nasceram e foram acompanhadas desde o nascimento até duas semanas ou mais de internação tiveram aumento no peso e comprimento, cerca de $37,1 \%$ e $51,4 \%$, respectivamente. Verifica-se que o crescimento dos recém-nascidos pré-termo foi satisfatório e que a utilização dos padrões de crescimento INTERGROWTH-21st, através de suas curvas antropométricas pós-natais, são úteis e de fácil aplicabilidade no diadia nas rotinas das unidades de terapia intensiva neonatais.
\end{abstract}

Palavras-chave: Intergrowth-21 ${ }^{\text {st }}$; Pré-termo; Recém-nascido.

\begin{abstract}
Use the postnatal growth curves of the INTERGROWTH-21st project for preterm newborns admitted to the Neonatal Intensive Care Unit of the Teaching Hospital of the Federal University of Pelotas-RS. Seventy newborns were followed up, with a prospective longitudinal design, from birth and / or hospitalization to discharge or death from the neonatal intensive care unit, from October 2018 to April 2019. Age estimated gestational age ranged from 19 to 36 weeks (mean 31 weeks), and $40 \%$ of newborns had birth weight less than $1500 \mathrm{~g}$, with $89 \%$ weighing less than 2500 g. Sixty preterm newborns were discharged and ten died during hospitalization - neonatal mortality of 143 per 10,000 live births. About $60 \%$ of births occurred by cesarean section, and 13\% of newborns had an Apgar score below 4 in the first minute of life. It was observed that through anthropometric measurements, both in boys and girls, during hospitalization, they were mostly within the normal limits of growth, and the growth percentiles of children who were born and were followed from birth until two weeks or more of hospitalization had an increase in weight and length, about $37.1 \%$ and $51.4 \%$, respectively. It appears that the growth of preterm newborns was satisfactory and that the use of the Intergrowth growth patterns, through their post-natal anthropometric curves, are useful and easy to apply in the daily routine of the unit neonatal intensive care.
\end{abstract}

Keywords: Intergrowth-21 ${ }^{\text {st }}$; Preterm; Newborn. 


\begin{abstract}
Resumen
Utilizar las curvas de crecimiento postnatal del proyecto INTERGROWTH-21st para recién nacidos prematuros ingresados en la Unidad de Cuidados Intensivos Neonatales del Hospital Docente de la Universidad Federal de Pelotas-RS. Se realizó un seguimiento de 70 recién nacidos, con un diseño longitudinal prospectivo, desde el nacimiento y / u hospitalización hasta el alta o muerte de la unidad de cuidados intensivos neonatales, de octubre de 2018 a abril de 2019. La edad gestacional estimada osciló entre 19 a las 36 semanas (media 31 semanas), y el 40\% de los recién nacidos tenían un peso al nacer inferior a $1500 \mathrm{~g}$, y el $89 \%$ pesaba menos de $2500 \mathrm{~g}$. Sesenta recién nacidos prematuros fueron dados de alta y diez murieron durante la hospitalización: mortalidad neonatal de 143 por cada 10.000 nacidos vivos. Aproximadamente el $60 \%$ de los nacimientos se produjeron por cesárea y el $13 \%$ de los recién nacidos tuvo una puntuación de Apgar inferior a 4 en el primer minuto de vida. Se observó que a través de medidas antropométricas, tanto en niños como en niñas, durante la hospitalización, se encontraban en su mayoría dentro de los límites normales de crecimiento, y los percentiles de crecimiento de los niños que nacieron y fueron seguidos desde el nacimiento hasta dos semanas o más de hospitalización tuvieron un aumento en el peso y la longitud, alrededor del $37,1 \%$ y $51,4 \%$, respectivamente. Parece que el crecimiento de los recién nacidos prematuros fue satisfactorio y que el uso de los patrones de crecimiento INTERGROWTH-21st, a través de sus curvas antropométricas postnatales, son útiles y fáciles de aplicar en la rutina diaria de las unidades, cuidados intensivos neonatales.
\end{abstract}

Palabras clave: Intergrowth- $21^{\text {st}}$; Pretérmino; Recién nacido.

\title{
1. Introdução
}

A prematuridade é um problema de saúde pública global, com 15 milhões de prematuros nascidos anualmente. É a principal causa de mortalidade neonatal e a segunda causa de mortalidade em crianças abaixo de cinco anos de idade, sendo definida como o nascimento anterior às 37 semanas completas de gestação podendo ser classificado, com base na idade gestacional em: extremamente prematuro (menos de 28 semanas), muito prematuro (28 a 32 semanas) e prematuro moderado à tardio (32 a 36 semanas) (Howson, et al., 2013; Who, 2018).

O nascimento pré-termo é o principal fator de risco para as infecções neonatais e contribui para o crescimento a longo prazo de crianças com deficiência e morbidade substancial, tais como deficiências cognitivas, motoras, visuais e de aprendizagem (Chang, et al., 2013). O crescimento de crianças nascidas pré-termo deve ser cuidadosamente monitorado, através de medidas antropométricas do peso, comprimento e perímetro cefálico. Estas medidas são de baixo custo, não invasivas e retratam o crescimento do recém-nascido conforme a sua idade (Chang, et al., 2013).

Para melhor avaliar o crescimento do recém-nascido pré-termo (RNPT) foi realizado entre 2006 e 2015 o projeto INTERGROWTH-21st, através do Consórcio Internacional do Crescimento Fetal e do Recém-Nascido para o Século XXI, uma rede multidisciplinar com mais de 300 médicos e cientistas de 27 instituições em 18 países no mundo. O componente de Acompanhamento Pós-natal do Pré-termo deste Projeto estabeleceu padrões de crescimento pós-natal em bebês prematuros (Villar, et al., 2015).

As unidades de terapia intensiva (UTI) neonatais e pediátricas surgiram no final na década de 1960 e vêm se desenvolvendo de forma constante, contribuindo significativamente para a melhoria dos cuidados de saúde e para a redução da mortalidade infantil intra-hospitalar. São nessas unidades que a maioria dos RNPT iniciam sua vida, necessitando de uma equipe de saúde capacitada para proporcionar os cuidados adequados (Barbosa \& Cunha, 2011).

A Unidade de Tratamento Intensivo Neonatal do Hospital Escola da Universidade Federal de Pelotas é classificada conforme habilitação do tipo II, com nove leitos disponíveis, sendo uma importante referência em neonatologia para a cidade de Pelotas e região (Ebserh, 2014). O Hospital Escola da Universidade Federal de Pelotas (HE-UFPEL), fundado em 1981, possui uma estrutura assistencial que integra ações de assistência ambulatorial e hospitalar de média e alta complexidade, presta atendimento a 22 municípios da região exclusivamente pelo Sistema Único de Saúde (SUS). É considerada referência na Zona Sul do Estado do Rio Grande do Sul/Brasil em diversas especialidades, com a adoção da prática humanizada tanto em cuidado em saúde, quanto na formação acadêmica (Ebserh, 2014).

Neste contexto, o presente estudo teve como objetivo utilizar as curvas pós-natais de recém-nascidos pré-termo, 
produzidas a partir da mensuração realizada pelo Projeto INTERGROWTH-21st, para avaliar o crescimento, dos RNPT internados na Unidade de Terapia Intensiva Neonatal do Hospital Escola da Universidade Federal de Pelotas-RS.

\section{Metodologia}

Trata-se de um estudo quantitativo, onde os dados foram aferidos e analisados por meio de técnicas estatísticas de caráter observacional longitudinal prospectivo (Pereira, et al., 2018), realizado para a utilização de instrumento de avaliação do crescimento de recém-nascidos pré-termo no período de outubro de 2018 a abril de 2019, na Unidade de Terapia Intensiva Neonatal (UTINEO) do Hospital Escola da Universidade Federal de Pelotas-RS (HE-UFPEL). A amostra foi realizada por conveniência dos RNPT internados, totalizando 70 acompanhamentos. Todos os RNPT foram elegíveis para o estudo, tendo sido excluídos os recém-nascidos à termo e os que necessitaram de reinternação.

O desfecho de interesse foi o acompanhamento do crescimento dos RNPT na UTINEO, através das medidas antropométricas peso, comprimento, perímetro cefálico, utilizando como Padrão as curvas INTERGROWTH-21st e a verificação dos padrões alimentares. Foram investigadas as seguintes variáveis independentes: sexo do RN (masculino ou feminino); cor da pele (branca, preta, parda, amarela, outra); idade materna (em anos); paridade materna (em número); tipo de parto (vaginal ou cesárea); Apgar (no primeiro minuto), estimativa da idade gestacional (IG) (em semanas), através de ultrassonografia (US), dia da última menstruação (DUM), método de Capurro, idade gestacional em semanas.

A coleta dos dados foi realizada desde o nascimento e/ou internação até a alta ou óbito do RNPT. Os prontuários foram observados diariamente, os tipos de alimentação Leite Materno (LM), Fórmula (F), Leite Materno e Fórmula (LM+F), Nada Por Via Oral (NPVO) e semanalmente as medidas antropométricas.

Os dados foram digitados através do programa Epidata 3.1 (Epidata, 2020) e analisados no software Statistical Package to Social Sciences for Windows V21.0 (Spss, 2020). Para as análises univariadas foram utilizadas as frequências absoluta e relativa. As medidas antropométricas observadas no estudo foram avaliadas através das curvas pós-natais de recémnascidos pré-termo através das ferramentas disponibilizadas no INTERGROWTH-21st (Intergrowth, 2020).

Os preceitos éticos previstos foram norteados conforme a resolução 466/2012 do Conselho Nacional de Saúde (CNS), do Ministério da Saúde, que dispõe a respeito das pesquisas com seres humanos. O estudo foi aprovado pelo Comitê de Ética em Pesquisa da Universidade Católica de Pelotas (UCPel): Parecer n ${ }^{\circ}$ 97776618.9.0000.5339 em 18 de outubro de 2018.

\section{Resultados e Discussão}

\subsection{Saúde ao Nascer e Dados Maternos}

Em relação as condições de saúde ao nascer e dados maternos dos 70 RNPT acompanhados no estudo, a idade gestacional estimada principalmente por ultrassom, variou de 19 a 36 semanas (média de 31 semanas), 40\% dos recémnascidos tiveram peso ao nascer inferior a $1500 \mathrm{~g}$, com 89\% apresentando peso inferior a $2500 \mathrm{~g}$. Sobre a alta da UTI Neonatal 60 RNPT tiveram, dez recém-nascidos faleceram durante a hospitalização, apresentando mortalidade neonatal de 143 por 10000 nascidos vivos. Cerca de 60\% dos nascimentos ocorreram por cesariana, 13\% dos recém-nascidos apresentaram escore de Apgar abaixo de 4 no primeiro minuto de vida. Entre as mães, 21\% tinham idade superior a 34 anos, $41 \%$ eram primíparas, e $74 \%$ eram de cor branca.

Alguns aspectos podem favorecer um parto prematuro, entre eles: a idade da gestante, cor da pele e a paridade (Oliveira, et al., 2018). A idade materna exerce influência na ocorrência de parto prematuro, mulheres adolescentes (menores de 20 anos) e com idade avançada (maiores de 34 anos) apresentaram associação com o parto prematuro (Oliveira, et al., 2016). 
Quanto ao tipo de parto, a cesariana aumenta a proporção de nascimentos prematuros. Vale ressaltar que o risco de manter a gestação deve ser cuidadosamente planejado, avaliando as necessidades do parto associados aos comprometimentos do nascimento prematuro (Oliveira, et al., 2016).

Os dados do relatório publicado pelo Fundo Internacional de Emergência das Nações Unidas para a Infância (UNICEF) apontam que quase 30 milhões de crianças em todo o mundo nascem prematuras, pequenas demais ou adoecem a cada ano (Who, 2018). No Brasil, estimasse que 323 mil crianças nasceram prematuras em 2018, correspondendo a 10,9\% dos nascimentos no país (Datasus, 2018). No Rio Grande do Sul em 2014, cerca de 16.450 (11,48\%) foram considerados prematuros, verificou-se a prevalência do pré-termo de $11,72 \%$ para os recém-nascidos do sexo masculino e de 11,46\% para o sexo feminino (Souza, et al., 2019).

$\mathrm{O}$ nascimento pré-termo é um dos principais fatores associados à mortalidade perinatal e/ou a danos imediatos ou tardios ao recém-nascido (RN). Cerca de 2,5 milhões de RN morreram, sendo quase dois terços devido a complicações de um parto prematuro (Who, 2018). Um indicador importante é o peso ao nascer na situação de saúde do RN, sendo o baixo peso ao nascer associado ao aumento da taxa de mortalidade durante o período perinatal. Os dados corroboraram com os de outro estudo, que evidenciou que a proporção de prematuros entre os bebês de baixo peso ao nascer teve um aumento marcante, de 45\% em 1982 para 60\% em 1993 e 67,3\% em 2004 (Oliveira, et al., 2016).

$\mathrm{O}$ peso ao nascer menor que $2500 \mathrm{~g}$ representa quatro vezes mais chances de estar associado ao nascimento prematuro, sendo, um fator de risco relacionado à morbimortalidade neonatal (Oliveira, et al., 2016). A relação existente entre a criança pré-termo e o peso ao nascer é inversamente proporcional, ou seja, quanto maior o grau de prematuridade, menor o seu peso (Varaschini, et al., 2015).

\subsection{Medidas Antropométricas}

Observou-se que em todos os grupos dos RNPT as médias de peso, comprimento e perímetro cefálico, tanto em meninos quanto em meninas, conforme o passar das semanas de hospitalização estiveram, em sua maioria, dentro dos limites normais de crescimento (percentil 3 a 97) das curvas pós-concepcionais INTERGROWTH-21st. Destes três casos ficaram abaixo do percentil 3 para as medidas antropométricas, dois do sexo masculino e um do sexo feminino. Dois RNPT estiveram com percentil abaixo de 3 ao nascer, um (menino) faleceu na primeira semana de acompanhamento, e a menina que na semana seguinte da hospitalização, evoluiu para alta da UTINEO. Um RNPT (menino) apresentou o percentil abaixo de 3 em todas as medidas antropométricas desde o nascimento até a quinta semana de internação.

Ao serem analisados os RNPT que estiveram hospitalizados por duas semanas ou mais após o nascimento, comparando com o percentil apresentado ao nascer, verificou-se aumento em cerca de $37,1 \%$ no percentil de peso e $51,4 \%$, no do comprimento. Se mantiveram estáveis em relação ao peso $22,8 \%$ e a respeito do comprimento $20 \%$.

Devido à gravidade do estado de saúde do RNPT, sendo impossibilitado a sua aferição das medidas do comprimento e perímetro cefálico aconteceram perdas do acompanhamento na quinta semana de internação no grupo de idade gestacional (IG) de 19 a 27 semanas. No grupo IG 34-36 semanas, do sexo feminino, não de teve acompanhamento do peso, do comprimento e do perímetro cefálico, a partir da terceira semana porque os RNPT tiveram alta hospitalar. À medida que passaram as semanas de internação houve a consequente diminuição do número de RNPT acompanhados, pois a maioria evoluiu para a alta da unidade.

O RNPT é mais suscetível a acometimentos de saúde devido as suas imaturidades e condições clínicas no período pós-natal (Da Rosa, et al., 2020). A imaturidade fisiológica afeta diversos órgãos e sistemas (Boyle, et al., 2017). Em relação as alterações ocorridas pelo parto prematuro destacam-se: dificuldades no sistema respiratório, distúrbios no sistema cardiovascular, alterações no desenvolvimento neural, dificuldade na regulação térmica e distúrbios gastrointestinais (Da 
Rosaet al., 2020).

O crescimento é caracterizado como um processo contínuo, resultante da interação complexa entre fatores genéticos, nutricionais, hormonais e ambientais (Bocca-Tjeertes, et al., 2011; Lima, et al., 2014). O nascimento pré-termo expõe a criança para uma condição de grande risco nutricional, visto que o terceiro trimestre de gestação é marcado por intenso crescimento intrauterino (Brasil, 2013). Observa-se uma proporção maior de pré-termo pequenos para idade gestacional (PIG) (peso abaixo do percentil 10) ao nascimento, em relação aos de tempo normal de gestação (Kang, et al., 2019).

O padrão de crescimento pós-natal no pré-termo em sua fase inicial corresponde a uma diminuição do peso na primeira semana de vida (Cardoso-Demartini, et al., 2011), com ganho ponderal médio de 25g/dia nas semanas subsequentes (Silveira \& Procianoy, 2019). A redução dos fluídos corporais em excesso e das reservas de gordura também ocorrem nos neonatos a termo, sendo mobilizadas para a produção de energia (Mezzacappa \& Ferreira, 2016; Gallardo López, et al., 2018).

A perda de peso é fisiológica quando corresponde até $20 \%$ do peso de nascimento do RNPT (Meireles, et al., 2018). A segunda fase, de transição, caracteriza-se pela estabilização de suas condições clínicas, assim o RNPT para de perder peso, sendo observado um discreto aumento do perímetro cefálico e do comprimento, bem como a recuperação do peso (CardosoDemartini, et al., 2011).

Geralmente a recuperação do peso de nascimento ocorre aproximadamente até o $14^{\circ}$ dia de vida (Sociedade Brasileira Pediatria, 2017). A terceira fase, ou fase de recuperação (catch-up), caracteriza-se por um aumento acelerado do crescimento com a finalidade de recuperação da fase inicial pós-nascimento. Espera-se que a aceleração máxima de crescimento ocorra entre 36 e 40 semanas de idade após a concepção e que a recuperação de medidas como o perímetro cefálico, comprimento e peso, ocorram até o primeiro ano de vida, entre 2 a 3 anos e até 3 anos, respectivamente (Cardoso-Demartini, et al., 2011).

\subsection{Alimentação dos Recém-Nascidos Pré-Termos}

No padrão alimentar dos RNPT, conforme o peso e as semanas de internação, individualizando a evolução para cada criança acompanhada, foi observado que a maioria dos que pesaram até 1000 gramas não conseguiram realizar alimentação enteral nos primeiros dias de vida, mas a partir da segunda semana em sua maioria o leite materno e a combinação leite materno e fórmula estiveram presentes na dieta. Nos RNPT de 1001-2000 gramas a maioria iniciou com fórmula, a dieta enteral e com o passar das semanas evoluiu para a combinação leite materno e fórmula. Já nos RNPT de 2001 gramas ou mais houve o predomínio de fórmula no início da dieta, mas em alguns casos a iniciação já se deu com leite materno, e com o passar das semanas evoluíram para a combinação leite materno e fórmula.

Alguns fatores podem influenciar o crescimento pós-natal de prematuros, tais como as intercorrências durante o período de internação e pós-alta hospitalar (Rover, et al., 2016), as práticas alimentares pós-natais (Damasceno, et al., 2014) e o estado nutricional ao nascimento (Lima, et al., 2014). A nutrição de boa qualidade é fundamental no crescimento do RNPT, sendo a má nutrição associada a desnutrição em nascidos pré-termo (Bocca-Tjeertes, et al., 2011; Alterman, 2016; Rover, et al., 2016).

O crescimento ideal está condicionado ao tipo, quantidade e qualidade da alimentação oferecida ao RNPT. A oferta calórica influencia no ganho de peso e a adequada utilização de proteínas está associada ao aumento do perímetro cefálico e do comprimento (Cardoso-Demartini, et al., 2011).

A avaliação nutricional em RNPT internado na UTI neonatal deve ser associada aos parâmetros antropométricos, bioquímicos, socioeconômicos e clínicos. Dessa forma é possível avaliar o seu estado nutricional de modo satisfatório adotando a conduta dietoterápica adequada para cada paciente (Pereira-Da-Silva, et al., 2019). 


\section{Conclusão}

A partir dos desfechos de interesse que o estudo se propôs, verificou-se que o crescimento dos RNPT foi satisfatório e que a utilização dos padrões de crescimento INTERGROWTH-21st, através de suas curvas antropométricas pós-natais, são úteis para essa mensuração e de fácil aplicabilidade no dia-dia das rotinas das UTINEO.

Destaca-se a importância de novos estudos abordando uma oferta alimentar de qualidade, predominantemente de leite materno, e a verificação contínua e sistematizada das medidas antropométricas. Estas ações são de baixo custo e proporcionam uma relevante forma de acompanhamento do desenvolvimento nos RNPT.

Como dificuldades na realização deste estudo, coloca-se a rotina de acompanhamento das medidas, pois são repetitivas, necessitando persistência e organização na averiguação dos dados. Acreditasse que seria interessante que trabalhos futuros avaliassem o tipo de dieta que será oferecida no momento da internação, associando esse dado ao crescimento pósnatal, nesta perspectiva entendesse que se poderia aperfeiçoar e otimizar parâmetros e com isso enriquecer o cuidado.

\section{Referências}

Alterman, C. S. (2016). Crescimento de recém-nascidos pré-termo de muito baixo peso durante internação em Unidade de Terapia Intensiva neonatal e fatores associados. http://repositorio.ufsm.br/handle/1/11273

Barbosa A. P., \& Cunha A. J. A. (2011). Terapia Intensiva neonatal e Pediátrica no estado do Rio de Janeiro, Brasil: uma análise de distribuição de leitos, 1997-2007, Caderno de Saúde Pública, 27. https://doi.org/10.1590/S0104-42302002000400035

Bocca-Tjeertes, I. F., Kerstjens, J. M., Reijneveld, S. A., \& De Winter, A. F. et al. (2011). Growth and predictors of growth restraint in moderately preterm children aged 0 to 4 years. Pediatrics, 128(5), 1187-1194. 10.1016/j.jpeds.2012.03.016

Boyle, A. K., Rinaldi, S. F., Norman, J. E., \& Stock, S. J. (2017). Preterm birth: Inflammation, fetal injury and treatment strategies. Journal of Reproductive Immunology, 119, 62-66. https://doi.org/10.1016/j.jri.2016.11.008

Brasil. (2012). Ministério da Saúde. Resolução CNS $N^{o}$ 466. Conselho Nacional de Saúde. Brasília, Distrito Federal, bvsms.saude.gov.br/bvs/saudelegis/cns/2013/res0466_12_12_2012.html.

Brasil. (2013). Ministério da Saúde. Secretaria de Atenção à Saúde. Departamento de Ações Programáticas Estratégicas. Atenção humanizada ao recém-nascido de baixo peso: Método Canguru: manual técnico/Ministério da Saúde, Secretaria de Atenção à Saúde, Departamento de Ações Programáticas Estratégicas. 2ed., Brasília: Editora do Ministério da Saúde. http://bvsms.saude.gov.br/bvs/publicacoes/atencao_humanizada_recem_nascido_canguru.pdf

Cardoso-Demartini, A. D. A., Bagatin, A. C., Silva, R. P. G. V. C. D., \& Boguszewski, M. C. D. S. (2011). Crescimento de crianças nascidas prematuras. Arquivos Brasileiros de Endocrinologia \& Metabologia, 55 (8), 534-540. https://doi.org/10.1590/S0004-27302011000800006

Chang, H. H, Larson, J, Blencowe, H, Spong, C. Y, Howson, C. P, \& Smith, S. C. et al. (2012). Preventing preterm births: analysis of trends and potential reductions with interventions in 39 countries with very high human development index. Lancet, 381, 223-234. http://dx.doi.org/10.1016/ S0140$6736(12) 61856-X$

Da Rosa, N. P., De Oliveira, D. C., Jantsch, L. B., \& Neves, E. T. (2020). Agravos agudos de saúde de bebês prematuros moderados e tardios no período neonatal. Research, Society and Development, 9 (7), 251974156-251974156. http://dx.doi.org/10.33448/rsd-v9i7.4156

Damasceno, J. R., SILVA, R. D., Ximenes Neto, F., \& Ferreira, A. et al. (2014). Nutrição em recém-nascidos prematuros e de baixo peso: uma revisão integrativa. Rev Soc Bras Enferm Ped, 14 (1), 40-46. https://journal.sobep.org.br/wp-content/uploads/articles_xml/2238-202X-sobep-S16763793201400007/2238-202X-sobep-S1676-3793201400007.x65337.pdf

Datasus. (2020). Nascidos vivos - Brasil: Nascimento por ocorrência por região segundo duração gestacional no ano de 2018. http://tabnet.datasus.gov.br/cgi/tabcgi.exe?sinasc/cnv/nvuf.def 2020

Ebserh. Empresa Brasileira de serviços Hospitalares. (2014). Diretoria de Atenção à Saúde e Gestão de Contratos Hospital Escola UFPEL, Brasília, Brasil. Dimensionamento de Serviços Assistenciais e da Gerência de Ensino e Pesquisa. http://www2.ebserh.gov.br>documents〉 dimensionamento

Epidata. (2021). All materials copyright by EpiData 3.1 Association 2000-2021 unless otherwise stated. Disclaimer and GNU license Revised Sat. https://www.epidata.dk/download.php

Gallardo López, M., Gallardo Cadenasso, E., \& Gallardo Cadenasso, L. (2018). Descenso de peso en recién nacidos a término en las primeras 48 horas post natales. Revista chilena de pediatría, 89 (3), 325-331. https://www.revistachilenadepediatria.cl/index.php/rchped/article/view/187

Howson, C. P., Kinney, M. V., Mcdougall L., \& Lawn J. E. (2013). Born Too Soon Preterm Birth Action Group. Born too soon: preterm birth matters. Reprod Health., 10 Suppl 1: S1. 10.1186/1742-4755-10-S1-S1

Intergrowth-2 $1^{\mathrm{ST}}$. (2020). The international fetal and newborn growth consortium for the $21^{\text {st }}$ century. https://intergrowth21.tghn.org/standards-tools 
Research, Society and Development, v. 10, n. 2, e9510212319, 2021

(CC BY 4.0) | ISSN 2525-3409 | DOI: http://dx.doi.org/10.33448/rsd-v10i2.12319

Kang, L., Wang, H. E. C., \& Wang, K. et al. (2019). Postnatal growth in preterm infants during the first year of life: A population-based cohort study in China. Plos one, 14 (4). https://doi.org/10.1371/journal.pone.0213762

Lima, P. A. T., Carvalho, M. D., Costa, A. C. C. D., \& Moreira, M. E. L. (2014). Fatores associados à restrição de crescimento extrauterino em recém-nascidos pré-termos de muito baixo peso ao nascer. Jornal de Pediatria, 90 (1), 22-27. Disponível em: https://doi.org/10.1016/j.jped.2013.05.007

Meireles, A. V. P., Oliveira, M. R. D. S., Nobre, R. G., \& Sousa, V. B. G. D. (2018). Assistência Nutricional em Neonatologia: Protocolo clínico Sistema de Gestão de qualidade 2018. www.ebserh.gov.br>documents> PRO.NTR.005+-+A

Mezzacappa, M. A., \& Ferreira, B. G. (2016). Perda de peso excessiva em recém-nascidos a termo amamentados exclusivamente ao seio materno em um Hospital Amigo da Criança. Revista Paulista de Pediatria, 34 (3), 281-286. http://dx.doi.org/10.1016/j.rppede.2016.03.003

Oliveira, L. L., Gonçalves, A. C., Costa, J. S. D., \& Bonilha, A. L. L. (2016). Maternal and neonatal factors related to prematurity. Rev Esc Enferm USP. 50 (3), 382-389.

http://dx.doi.org/10.1590/S0080-623420160000400002

Oliveira, K. A., Araújo, E. M., Oliveira, K. A., Casotti, C. A., Silva, C. A. L., \& Santos, D. B. (2018). Associação entre raça/cor da pele e parto prematuro: revisão sistemática com meta-análise. Revista de Saúde Pública, volume 52 (26). https://doi.org/10.11606/s1518-8787.2018052000406

Pereira, A.S. et al. (2018). Metodologia da pesquisa científica. Ed. https://repositorio.ufsm.br/bitstream/handle/1/15824/Lic_Computacao_Metodologia-Pesquisa-Cientifica.pdf?sequence=1

Pereira-da-Silva, L., Virella, D., \& Fusch, C. (2019). Nutritional Assessment in Preterm Infants: A Practical Approach in the NICU. Nutrients, 11 (9). https://doi.org/10.3390/nu11091999

Rover, M. M., Viera, C. S., Silveira, R. C., \& Guimarães, A. T. et al. (2016). Risk factors associated with growth failure in the follow-up of very low birth weight newborns. Jornal de Pediatria, 92 (3), 307-313. http://dx.doi.org/10.1016/j.jped.2015.09.006

Silveira, R. C., \& Procianoy, R. S. (2019). Preterm newborn's postnatal growth patterns: how to evaluate them. Jornal de pediatria, 95 (2), 42-48. 10.1016/j.jpedp.2018.10.004

Sociedade Brasileira Pediatria. (2017). Monitoramento do crescimento de RN pré-termos. Departamento Científico de Neonatologia. www.sbp.com.br fileadmin > user upload > 2017/03

Souza, D. M. L, Maia, L. C. S., Zêgo, Z. D. F., Jaeger, G. P., \& Maciel, W. S. (2019). Prevalência de prematuridade e fatores associados no estado do Rio Grande do Sul. Brazilian Journal of health Review, Curitiba, 2 (5), 4052-4070. 10.34119/bjhrv2n5-014

Spss. (2020). Statistical Package to Social Sciences for Windows V21.0. https://www.ibm.com/support/pages/spss-statistics-210-available-download

Varaschini, G. B., Molz, P., \& Pereira, C. S. (2015). Perfil nutricional de recém-nascidos prematuros internados em uma UTI e UCI neonatal. Cinergis, 16 (1), 05-08. http://dx.doi.org/10.17058/cinergis.v16i1.5137

Villar, J., Giulianni, F., Bhutta, Z. A., Bertino, E., Ohuma, E. O., \& Ismail, L. C., et al. (2015). Postnatal growth standards for preterm infants: The Preterm Postnatal Follow-up Study of the Intergrowth-21(st) Project. Lancet Glob Health. 3 (11), 681-691. 10.1016/S2214-109X (15)00163-1

Who. (2018). World Health Organization. Survive and thrive: transforming care for every small and sick newborn. https://www.who.int/maternal_child_adolescent/documents/care-small-sick-newborns-survive-thrive/en/ 